\title{
Design and development of liquid filled polymeric fibers with flexure rate viscoelastic properties
}

\author{
L. Gottardo ${ }^{1 *}$, R. Hufenus ${ }^{1}$, M. Dressler ${ }^{2}$ and M. Heuberger ${ }^{1}$ \\ ${ }^{1}$ EMPA, Swiss Federal Laboratories for Materials Science and Technology, Lerchenfeldstrasse 5, 9014-CH St. Gallen \\ -laura.gottardo@empa.ch; rudolf.hufenus@empa.ch; manfred.heuberger@empa.ch \\ ${ }^{2}$ Department of Chemical Engineering, University of Massachusetts Amherst, Amherst, MA, U.S.A. \\ -dressler@ecs.umass.edu
}

\begin{abstract}
The design and development of a novel category of bi-component polymeric fibers, made of a polymeric sheath and a liquid core is described. To understand and to analyze the flow of molten polymer and liquid within a spin pack, we built a macroscale analogon where co-flow of polymer and liquid is investigated systematically. The macroscale analogon is composed of a glass cylinder with a finite length and a coaxial capillary. The inner fluid flows in the capillary and at its outlet meets the outer fluid flowing inside the glass cylinder. The fluids are pumped into the setup using pressurized bottles with controlled flow rates. A high-speed camera is placed in front of the glass cylinder to record the transient and the steady flow of the two phases and the morphology of their interface during co-flow. We systematically analyzed the combination of various fluids and different injection channel geometries, and used these findings to design a customized spin pack. Extrusion trials with molten polymers and specific liquids resulted in the production of liquid filled polymeric fibers (LFF) with different amounts of liquid from $3 \%$ to $33 \%$ vol. and diameters from $150 \mu \mathrm{m}$ to $1 \mathrm{~mm}$. Fiber morphology has been investigated with the help of optical microscopy and computer tomography, in order to analyze both surface and core morphology. Mechanical characterization of fibers has been carried out with special care to the damping capacity of such fibers using two different methods: two point bending tests to evaluate the energy dissipation of fibers upon bending at low frequency values $(0.005-0.5 \mathrm{~Hz})$, and analysis of vibration reduction to determine the quality factor at the resonance frequency of the $1^{\text {st }}$ and $3^{\text {rd }}$ vibration mode.
\end{abstract}

Keywords: Co-flowing, co-extrusion, liquid filled fibers, energy dissipation, damping.

\section{INTRODUCTION}

Nowadays the combination of different materials in one single bicomponent fiber is a well-established technique. With this method, special qualities and structures are given to the fibers and their textile products. Usually the components are synthetic polymers, however metals, carbon and cellulose are examples of other components used. Due to the various ranges of application the importance of bicomponent fibers is steadily growing $[1,2]$. Our work is focused on the production of a new kind of bicomponent fiber, composed of a polymeric sheath and a liquid core using the melt-spinning technique. In this paper we describe methods to design and produce such fibers. In the first part we present the results of an investigation performed using an experimental visualization set up built in order to mimic a real spin pack. Experimental results as well as numerical modeling (not presented in this paper) allowed us to design a melt-spinning die which enables the co-flowing of a molten polymer and a liquid. In the second part the co-extrusion experiments and the obtained fibers are described. The third part is devoted to fiber characterization, both morphological and mechanical, with a special focus on damping properties. We believe that this novel category of fibers can open a wide range of applications. The presence of the inner liquid, if properly chosen and interconnected with the polymer sheath, can give fibers shock absorbing properties, motion damping or high speed responsive capacity. For example, such fibers could find application as replacement of actual protective garments which rely on flexible materials [3-5]. Outlook of this research are further optimization of processing steps as well as a new choice of material combination.

\section{EXPERIMENTAL}

\section{Experimental visualization set up}

The experimental set-up is shown in Fig. 1a. Fluids are stored in two glass bottles (volume $500 \mathrm{ml}$ ) which are pressurized with a compressed air source of 6bar. The inlet pressure is controlled by pressure reducers (SMC, IR2000 F02; NORGREN, 11-818-999) with a range of outlet pressures between 0.02 and 2 bar. The fluids are pumped through flexible tubes to the capillary system consisting of an outer glass tube and a a coaxial inner stainless needle (Hamilton, G11). The co-axiality of outer tube and needle is assured by a Swagelock "Tee" connection (SS-10M0-3-10M-6M). We used different kinds of injection needles: a standard one without holes (labeled $N_{0}$ ) and needles with two and four holes (labeled $N_{2}$ and $N_{4}$, respectively). The holes are distributed equally along the circumference of the capillary, i.e. every $180^{\circ}$ for the two-hole needle, and every $90^{\circ}$ for the four-hole needle. Schematics of the three needles and are shown in Fig. 1b. 


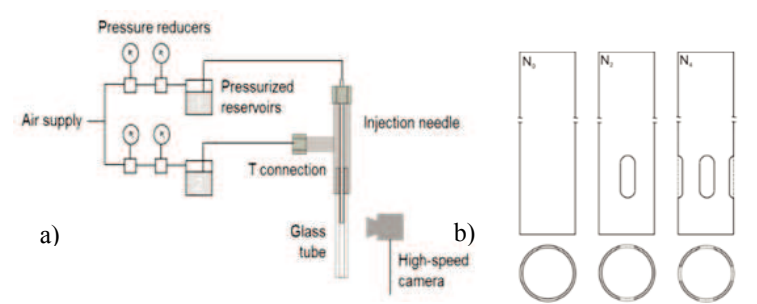

FIGURE 1 - Schematics of a) experimental visualization set up; b) injection needles.

\section{Co-extrusion set up}

Fig. 2 shows the spin pack enabling the co flow of a molten polymer and a liquid.

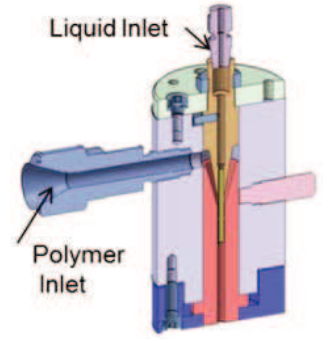

FIGURE 2 - 3D cross sectional view of the melt spinning die used to make co-extrusion exeriments.

Polymer is supplied by a twin screw extruder and a metering gear pump to control the mass flow rate, while the liquid is pumped into the device using a piston pump operating with constant flow. Molten polymer and liquid are flowing together in the co-flow channel until they reach the outlet forming an extrudate. This is subsequently cooled down in a water bath and drawn using three heatable godets before being collected on a spool.

\section{Materials}

In the experimental visualization set up, water and an ethanol aqueous solution $(25 \% \mathrm{vol})$ have been used as inner fluids, vaseline oil (GPR Rectapur, VWR) and different viscosity grades of polydimethylsiloxane (Xiameter, PMX200, 50-100-200-300 cS) have been used as outer fluids. All visualization experiments have been performed at room temperature. Different kind of polymers have been used for co-extrusion: polypropylene (Sabic PP621P, PPPCGR25), low density polyethylene (Sabic, 1965ST), polyamide 6 (EMS, Grilon F34NL) and polyamide 12 ( EMS, Grilamid L16). As liquids: polydimethylsiloxane (Xiameter, PMX 200 - 300 and 2000 cS), polyaphaolefin oligomers (Ineos, Durasyn 180L; ILCO Chemicalien, ILCO lube 7030) polyalkenglicol (BASF, Breox 75W 2050) and polyethylenglycol (Sigma Aldrich, PEG600).

\section{RESULTS AND DISCUSSION}

\section{Example of two phase liquid morphology}

In our experimental analysis, we investigated the effects of a) flow rate ratio, b) interfacial tension, c) viscosity ratio, and d) lateral permeability of the injection needle, on the observed interfacial characteristics. We classify the observed flow/interfacial morphologies as "droplets", "slugs", "(stable) core annular flows", and "unstable core annular flows" as illustrated in Fig. 3 as a function of flow rate ratio.

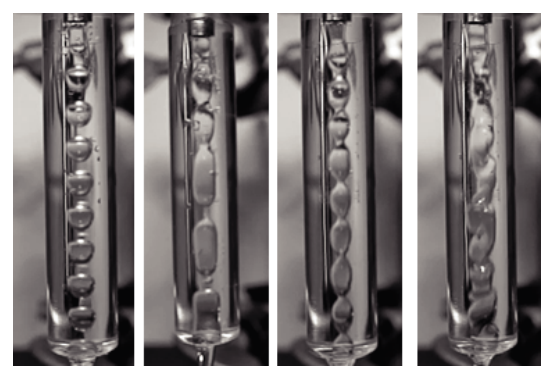

FIGURE 3 - Examples of two phase morphology: droplets, slugs, core annular flow, and unstable core annular flows (from left to right).

Comparing the results of the two series of experiments performed with distilled water and aqueous ethanol solution as dispersed phases, we could underline the effect of the interfacial tension on the interface morphology. In fact, the surface tensions of water and ethanol solution are $70 \mathrm{mN} / \mathrm{m}$ and $48 \mathrm{mN} / \mathrm{m}$ respectively, and their density differs of less than $5 \%$. Therefore the parameter responsible of the transition from a dripping to a jetting regime can be identified in the different interfacial tension between the two fluids (Fig 4).

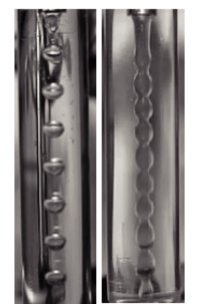

FIGURE 4 - Pictures showing the effect of interfacial tension on interface morphology: water as dispersed phase dripping regime (left); ethanol solution as dispersed phasejetting regime (right).

Dripping results from interfacial tension, which forces the droplet to adopt a spherical shape when it is formed at the capillary tip. When the interfacial tension decreases, this effect becomes less pronounced and a long thinning jet can be observed. We could clearly find this effect in our experiments, as shown in Fig. 4. One can assume that the dripping to jetting transition occurs at lower velocity of the continuous phase. This is in good agreement with what has been observed in [6], where droplet break up in dripping regime has been studied by changing the most relevant parameters, and correlating the droplet size with the flow conditions.

Fig. 5 shows how different viscosity grades result in totally different morphologies under identical flow rates conditions, i.e. inner flow rate $Q_{l}=1.27 \mathrm{ml} / \mathrm{s}$ and outer flow rate $Q_{2}=0.49 \mathrm{ml} / \mathrm{s}$. 


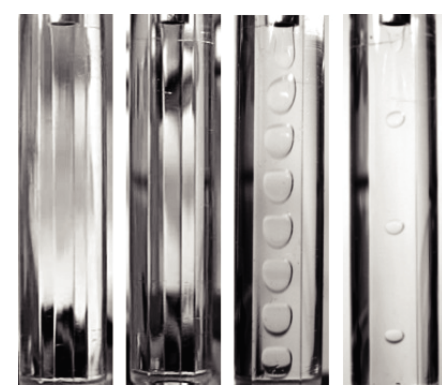

FIGURE 5 - Snapshots of high speed movies obtained with PDMS of different viscosities using $1.27 \mathrm{ml} / \mathrm{s}$ as inner flow rate $Q_{1}$ and $0.49 \mathrm{ml} / \mathrm{s}$ as outer flow rate $Q_{2}$. From left to right site the outer phase viscosities are 50,100, 200 and $300 \mathrm{cS}$ respectively.

For the different flow rate ratios examined, we were able to observe periodic modulations of the jet diameter, but neither Rayleigh instability, nor surface waves propagating down-stream and leading to jet break-up were detected. When the viscosity of the continuous phase was increased, while flow rate ratio and injection needle geometry remained unchanged, the interfacial morphologies changed from continuous jets over slugs to droplets. This means that a continuous phase with low viscosity exerts a small tangential force on the freely flowing disperse phase. Consequently a specific flow rate of the dispersed phase is required to lead to the formation of a continuous jet. If instead the viscosity of the disperse phase is increased, the shearing force opposing free flow of the disperse phase rises, which in combination with the surface forces, is prone to the formation of droplets or slugs, depending on the flow rate conditions hindering the generation of a jet. The effect of the lateral permeability of the injection needle is summarized in Fig. 6; in particular, Fig. 6a shows large droplets with an irregular, non-spherical shape, Fig. 6b and $\mathrm{c}$ show comparably small droplets of ellipsoidal shape. Furthermore, note that droplets in Fig.6b and c are flattened and mutually touching. We believe that the van-der-Waals forces between these droplets are comparable to the interfacial forces, and need to be taken into account in order to understand the interfacial morphologies seen in Fig.6b and c. When the capillary needle with two or four side holes is used, we find that droplets emerge from the injection needle with smaller diameter and higher frequency as compared to the standard needle without side-holes. In both cases droplets are elongated in the radial direction and under flow they shift away from the center-line. This implies that in this section of the flow the intra-droplet (i.e. interfacial tension) forces become more relevant than the attractive inter-droplet (i.e. van der Waals) forces.

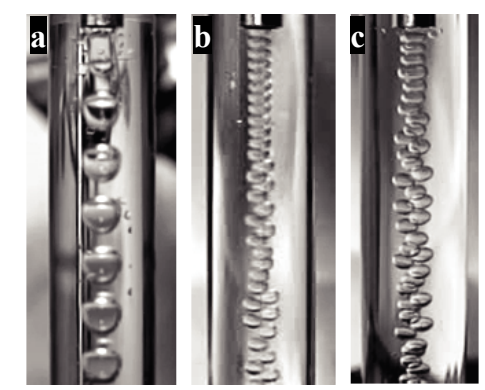

FIGURE 6 - Snapshots of high speed recorded movies obtained with vaseline oil and distilled water as outer and inner fluid, respectively, using the three different needle geometries N0, N2 and N4, respectively (from left to right). Flowing conditions are $Q_{1}=1.75 \mathrm{ml} / \mathrm{s}$ and $Q_{2}=0.49 \mathrm{ml} / \mathrm{s}$ for inner and outer fluids.

Production and characterization of liquid filled fibers Different types of fibers have been produced using a variety of material combinations, processing parameters and spinneret geometries, with the goal to reproduce the morphologies observed in the experimental set up. The spinning pack was built with the same geometry and dimensions of the experimental set-up. Processing parameters have been chosen based on the results of the previous observation with the experimental set-up and of numerical simulations. Our results show that fibers can basically contain a variable part of liquid between $3 \%$ and $33 \%$. The fiber diameters realized were between one millimeter down to a few hundred microns. The large range of fiber diameters produced can be achieved due to two distinct characteristics of the process: i) the newly designed spinning pack overtakes large polymer flows, which allows the combination with large volumetric flow rates of the liquid, and ii) the high efficiency of the drawing process which leads to a large diameter reduction. It is interesting to note that above a critical amount of liquid, melt-spinning and fiber drawing became impossible. This is inevitable due to an excessive thinning of the polymeric sheath, which embodies the liquid thread. A morphological characterization of liquid filled fibers has been performed using computer tomography (CT). Due to rather clear differences in X-ray absorption one can easily distinguish the polymer phase from the liquid one and compute a 3D picture of a sample. As an example, three fibers with inner liquid droplets are shown in Fig. 7a. This reveals how the dimension of the droplets and their frequencies can be varied.

LFF with a continuous core exhibit no significant longitudinal diameter variations (Fig. 7b). If we draw a cross section from the CT scan, we find that the liquid channel readily reveals a non-circular perimeter and the center of weight of the core may be off the fiber axis (Fig. 7c). 

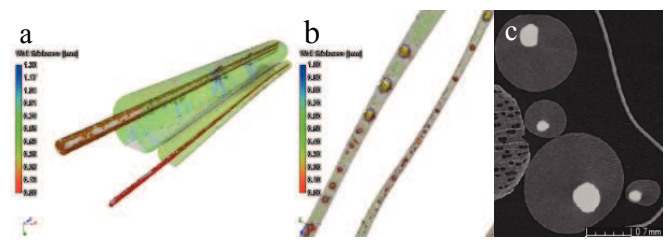

FIGURE 7 - Micro CT pictures of : a) fibers with continuous liquid core; b) fibers with liquid droplets; c) fibers cross section.

A systematic core eccentricity might be due to minute pressure gradients inside the spin die where polymer is, for practical reasons, only from side inside the die. Another effect that amplifies eccentricity is the lower viscosity of the liquid compared to the molten polymer. This instability (c.f. fingering) gives rise to noncircular protrusions and off axis migration.

We characterized the mechanical properties of LFF with particular attention on their capability to absorb energy, i.e. their damping properties. Therefore we used two methods: two point bending test and analysis of vibration decay. The first method provides information on the quasi-static bending response of fibers, the second gives information about the quality factor of the fiber which is directly related to its damping properties. In Fig. 8 the force necessary to bend a LFF is shown for three cycles of loading/ unloading, and it is compared to the force necessary to bend a pure polymeric fiber (reference) of the same dimension. The hysteresis between loading/unloading yields the amount of dissipated energy upon bending, i.e. the capacity of the fiber to dissipate energy upon deformation. It can be calculated that during the first cycle a LFF can dissipate $21 \%$ of the energy, in comparison to only $5.8 \%$ of a standard fiber.

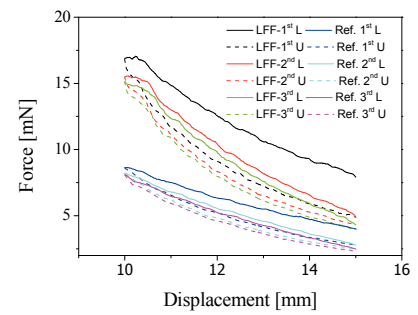

FIGURE 8 - Loading/unloading cycles for LFF and pure polymer fiber (reference).

Using the vibration decay method, a fiber was kept in tension within two clamps and was excited at its resonance frequency using a loudspeaker. With a laser vibrometer the change of velocity of the vibration of the fiber was measured and the quality factor $\mathrm{Q}$ could be determined according to Eq. 1 .

$Q=\frac{f_{\text {res }}}{\Delta f}$

With $f_{\text {res }}$ resonance frequency of the vibration and $\Delta f$ the bandwith of frequency at $-3 \mathrm{~dB}$ lower than the maximum ampitude at the resonance frequency. If energy (in the form of acoustic waves) is supplied to the fiber, it will vibrate but the vibrations will gradually die away because of damping. In this case, damping is a measure of how rapidly the fiber loses energy through friction or by radiating the energy away as sound, heat etc. If the damping is weak, the vibrations will continue for a long time, therefore the $\mathrm{Q}$ factor will be high. If the damping is strong, the vibrations will die away quickly, therefore $\mathrm{Q}$ factor will decrease. In Fig. 9 it is seen that fibers filled with a low visocus liquid are those who have better damping properties compared to standard polymeric fibers, or to fibers filled with a high viscous fluid.

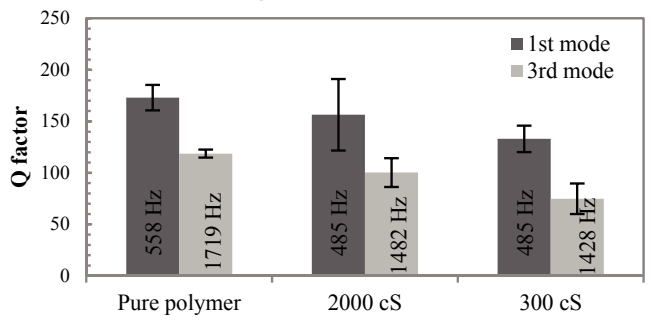

FIGURE 9 - Q factor of a pure polymer fiber, a high viscous fluid $(2000 \mathrm{cS})$ filled fiber and a low visocous fluid $(300 \mathrm{cS})$ filled fiber.

\section{CONCLUSION}

With this work we demonstrate the capability to produce liquid filled fibers continuously in a one step process. Different polymer and liquid combinations could successfully be produced to fibers with a large variety of liquid amount and dimension size. Further improvement can be achieved by optimizing the process and looking for a more interconnected structure between liquid and polymer. In this way fibers could sustain more efficient damping properties than those showed up to now. Moreover by using shear thickening fluids in the core, we could obtain an adaptive response as a function of the flexural bending rate, giving additional smart characters to such fibers.

\section{ACKNOWLEGDEMENTS}

Authors thank Swiss National Science Foundation NRP62 "Smart Materials" for the financial support.

\section{REFERENCES}

1. Houis, S., F. Schreiber, and T. Gries, Bicomponent fibers (Part 2) 2nd issue 2008. Chemical Fibers International, 2008. 58(3): p. 158-165.

2. Houis, S., F. Schreiber, and T. Gries, Fiber table: Bicomponent fibers (Part 1). Chemical Fibers International, 2008. 58(1): p. 38-45.

3. Budden, G., Defense and Comfort: New Advancement in Impact-Protection Textiles. T3 Technical Textiles Technology, 2006 (April).

4. Lee, Y.S., E.D. Wetzel, and N.J. Wagner, The ballistic impact characteristics of Kevlar $(R)$ woven fabrics impregnated with a colloidal shear thickening fluid. Journal of Materials Science, 2003. 38(13): p. 2825-2833.

5. Palmer, R.M. and P.C. Green, Energy absorbing material. 2007. p. 7.

6. Cramer, C., P. Fischer, and E.J. Windhab, Drop formation in a co-flowing ambient fluid. Chemical Engineering Science, 2004. 59(15): p. 3045-3058. 